\title{
RELACIÓN ENTRE EL PH SALIVAL Y LA PREVALENCIA DE CARIES DENTAL EN ESCOLARES DE 6 A 12 AÑOS DE LA INSTITUCIÓN EDUCATIVA SAN GABRIEL, VILLA MARÍA DEL TRIUNFO, 2017
}

\author{
RELATIONSHIP BETWEEN SALIVARY PH AND PREVALENCE OF DENTAL CARIES IN \\ SCHOOLCHILDREN FROM 6 TO 12 YEARS OF AGE AT THE SAN GABRIEL EDUCATIONAL \\ INSTITUTION, VILLA MARIA DEL TRIUNFO, 2017 \\ Carlos Alberto Araujo Muro ${ }^{1}$ \\ carauj0210@gmail.com \\ ORCID: 0000-0001-5143-7737
}

\section{RESUMEN}

Objetivo: Determinar la relación entre el $\mathrm{pH}$ salival y la prevalencia de caries dental en escolares de 6 a 12 años de la ciudad de Lima. Metodología: Estudio transversal desarrollado en 129 escolares de la institución educativa San Gabriel, del distrito de Villa María del Triunfo, en 2017. Se obtuvieron las mediciones del $\mathrm{pH}$ salival en los pacientes mediante el uso del pH-metro, asistido por tiras de papel y se evaluó la presencia de caries dental mediante el método CPO-D. También se evaluaron las características de sexo y edad de todos los pacientes. La estadística emplea las pruebas de Chi-cuadrado y se trabajó a un nivel de significancia de $p<0,05$. Resultados: Se encontró una prevalencia de caries dental del $85,3 \%$ en escolares entre los 6 y 12 años. La prevalencia de caries según el género fue del $45,7 \%$ en niñas y el $39,5 \%$ en varones. No se encontró asociación estadísticamente significativa entre el género y la prevalencia de caries dental $(p=0,935)$. El $55 \%$ del grupo muestral presentó un pH ácido; el
$41,1 \%$, un $\mathrm{pH}$ neutro, y el $3,9 \%$, un $\mathrm{pH}$ alcalino. Se determinó la existencia de una asociación estadísticamente significativa entre $\mathrm{pH}$ salival y caries dental $(\mathrm{p}$ $<0,001)$. Conclusiones: Existe una relación directa entre el $\mathrm{pH}$ salival y la prevalencia de caries dental. Se calculó que el $95,8 \%$ de los pacientes con un $\mathrm{pH}$ salival ácido presentaron caries dental.

Palabras clave: caries dental, escolares, $\mathrm{pH}$ salival

\section{ABSTRACT}

Objective: To determine the relationship between the salivary $\mathrm{pH}$ and the prevalence of dental caries in school children between the ages of 6 and 12 in the city of Lima. Methods: Transversal study of prevalence carried out on 129 schoolchildren from the "San Gabriel" Educational Institution from the district of Villa Maria del Triunfo, in the year 2017. The salivary $\mathrm{pH}$ measurements were obtained using the $\mathrm{pH}$-meter with the respective

Citar como: Araujo C. Relación entre el pH salival y la prevalencia de caries dental en escolares de 6 a 12 años de la institución educativa San Gabriel, Villa María del Triunfo, 2017. Rev Cient Odontol (Lima). 2019; 7 (2): 23-32. 
test-strips, and the presence of caries was evaluated in patients utilizing the CPO-D method. In addition, the sex and age characteristics were evaluated in all the patients. The statistics employed the Chi-squared test and we worked at a level of significance of $p<0.05$. Results: The prevalence of dental caries found in schoolchildren between 6 and 12 years old is $85,3 \%$. The prevalence of caries in accordance to gender was $45,7 \%$ amongst girls and 39,5\% amongst boys. No statistically significant association was found between gender and the prevalence of dental caries ( $p=0,935)$. $55 \%$ of the sample group exhibited an acidic $\mathrm{pH}$, while $41,1 \%$ had a neutral $\mathrm{pH}$, and $3,9 \%$ an alkaline $\mathrm{pH}$ respectively. There is a statistically significant association between the salivary $\mathrm{pH}$ and dental caries. $(p<0,001)$. Conclusions: There's an existing association between salivary $\mathrm{pH}$ and the prevalence of dental caries. It was calculated that $95,8 \%$ of the patients with an acidic salivary $\mathrm{pH}$ presented dental caries.

Keywords: dental caries, schoolchildren, salivary $\mathrm{pH}$

\section{INTRODUCCIÓN}

De todas las patologías que afectan la cavidad oral, la caries dental es la enfermedad crónica más común tanto en países desarrollados como en países en vías de desarrollo. Es una enfermedad que no discrimina, ya que está presente sin importar el sexo, el nivel socioeconómico, la raza o la edad ( $\left.{ }^{1}\right)$.

Se presenta cuando existe un desequilibrio en los procesos de remineralización y desmineralización del esmalte dental. Este balance depende de varios factores, principalmente del $\mathrm{pH}$ salival. En el caso de un $\mathrm{pH}$ salival ácido, el balance se inclina hacia la desmineralización, lo que genera una degradación tanto de la superficie como de la subsuperficie del esmalte. Este desbalance perturba la pieza dentaria en su conjunto, afectándola a nivel bioquímico, morfológico, funcional y sensorial $\left({ }^{1}\right)$. Recientes esfuerzos por descifrar los genomas bacterianos vinculados con la aparición de lesiones cariosas nos han llevado a comprender lo excepcionalmente diverso que es el ecosistema de la cavidad oral. Hoy sabemos que el Streptococcus mutans, una bacteria relacionada durante décadas con la caries dental, está ausente al inicio de la enfermedad y que existe un gran número de microorganismos similares que aparecen en diferentes etapas de la maduración de la placa dentaria $\left({ }^{2}\right)$.

La prevalencia de caries depende de una serie de factores, los cuales han ido aumentando con el paso de los años. Aparte de la conocida triada, que incluye la ya mencionada placa dental bacteriana, el consumo de carbohidratos y la susceptibilidad de las piezas dentarias, existen otros factores contribuyentes como la higiene oral y la calidad salival $\left({ }^{3,4}\right)$. De estos, la calidad salival depende de su composición y es considerada un factor de suma importancia para determinar la prevalencia de caries. La saliva tiene diversas características que determinan su calidad, tales como el $\mathrm{pH}$, la capacidad amortiguadora, la viscosidad y la tasa de flujo ${ }^{(5-7)}$. Entre estas, el $\mathrm{pH}$ es considerado determinante en la prevalencia de caries $(8,9)$. Sin embargo, no existen estudios actualizados que demuestren la relación entre el pH salival y la prevalencia de caries en niños de edad escolar en el Perú.

El mal estado de las piezas dentarias tiene un grave impacto en la salud general de cualquier ser humano, y es mayor aún en la población de este sector etario. Puede llegar a comprometer el crecimiento, estado nutricional y peso corporal debido a las molestias/dolores producidas por la 
presencia de caries dental, que pueden generar dificultades para dormir, desórdenes de aprendizaje e inasistencias a la escuela. Es más, las infecciones odontogénicas como consecuencia de una caries dental son el motivo de hospitalización más frecuente para niños en edad escolar. Es por estas razones que la salud oral de un niño es determinante al evaluar su estado general de salud y calidad de vida. Además, la presencia de caries en la dentición primaria podría generar un alto riesgo de caries para las demás piezas o fomentar la aparición de defectos en el desarrollo del germen de la pieza permanente $\left({ }^{10}\right)$.

La edad escolar se caracteriza por una dieta en la que predominan los alimentos con un $\mathrm{pH}$ ácido crítico, que aumenta directamente el riesgo de caries. Es responsabilidad de los padres llevar un control adecuado de lo que consumen sus hijos $\left({ }^{11}\right)$. Existen estudios que han demostrado fehacientemente que un $\mathrm{pH}$ crítico, es decir, un $\mathrm{pH}$ ácido menor a 5,5 favorece la formación de caries. Este fluido biológico es clave para mantener el equilibro del $\mathrm{pH}$ durante los procesos de desmineralización y remineralización que se producen en las piezas dentales $\left({ }^{12,13}\right)$.

La saliva cumple un papel crucial en la salud oral. Según su composición, adopta una serie de propiedades como lubricación, limpieza, digestión, acción amortiguadora y acción antimicrobiana. Asimismo, puede afectar la prevalencia de caries de 4 formas: como agente de limpieza mecánico; al reducir la solubilidad del esmalte dentario mediante el calcio, fosfato y flúor; al neutralizar los ácidos producidos tanto por organismos cariogénicos como los introducidos directamente mediante la dieta, y mediante su acción antibacterial $\left.{ }^{14-16}\right)$.

Otras investigaciones evaluaron la prevalencia de caries con relación al pH salival y han demostrado que existe una relación directa entre ambos. Los pacientes con un $\mathrm{pH}$ salival ácido muestran una mayor prevalencia de caries $\left.{ }^{(17-19}\right)$. Sin embargo, en el Perú, la investigación más reciente sobre esta relación tiene más de 5 años. En vista del vacío existente, este estudio buscará determinar la situación actual que enfrentamos con la finalidad de establecer políticas preventivas de salud efectivas en concordancia con nuestra realidad.

\section{MATERIALES Y MÉTODOS}

El método de la investigación fue la observación estructurada. La población estuvo conformada por los niños matriculados en la institución educativa San Gabriel, del distrito de Villa María del Triunfo, en 2017. La muestra estuvo compuesta por 129 niños. El tamaño de muestra fue determinado de acuerdo con un muestreo estratificado por conveniencia.

\section{MÉTODO}

Se ejecutó un estudio piloto con la finalidad de constatar la eficiencia de la metodología, determinar el tamaño muestral requerido y calibrar al examinador con el Gold Standard. Durante el proceso de calibración, se abordaron las normas teóricas y prácticas para el diagnóstico de caries dental según las bases establecidas por la AAPD, con lo que se logró unificar y estandarizar los criterios con relación a un Gold Standard. La calibración interexaminador se ejecutó mediante 5 pruebas por examinador, kappa $=0,8$.

Etapa 1. Se recolectaron las muestras de niños de 6 a 12 años pertenecientes a la institución educativa según los criterios de selección y exclusión. El examen se llevó a cabo previa autorización de los padres. Se redactó un documento de consentimiento informado en el que se detallaron los riesgos y beneficios que 
conlleva el presente estudio. Los niños cuyos padres no firmaron el consentimiento fueron excluidos del estudio.

Etapa 2. Examen clínico. Se procedió a realizar el diagnóstico de caries en un ambiente adecuadamente iluminado, empleando una sonda periodontal WHO Probe 11.5 y un espejo bucal $n .{ }^{\circ} 3$ con magnificación. Se utilizó el método de diagnóstico visual táctil, de acuerdo con las normas establecidas por la OMS. Se usó el índice de CPO-D. La presencia de manchas blancas activas en superficies lisas se consideró como caries dental. Subsecuentemente, se realizó la medición del $\mathrm{pH}$ utilizando un kit de $\mathrm{pH}$ (Panpeha) que contiene tiras reactivas y una guía de color correspondiente que nos indica la acidez o la alcalinidad de la saliva. La tira reactiva se colocó en la cavidad oral por 10 segundos y se procedió con la lectura.
Etapa 3. Los resultados fueron recopilados en una base de datos y se realizaron las pruebas estadísticas correspondientes. Una vez obtenido los resultados, se procedió con la interpretación y la redacción del informe final.

\section{RESULTADOS}

Se determinó la frecuencia de las variables caries, sexo y grupo etario mediante un análisis univariado. El análisis bivariado entre las variables, $\mathrm{pH}$ salival y prevalencia de caries dental se realizó mediante la prueba no paramétrica de Chi-cuadrado. Todo análisis estadístico se ejecutó con el programa SPSS versión 20.

Se evaluó a un total de 129 niños, el $53,5 \%$ del sexo femenino y el $46,5 \%$ del sexo masculino, entre los cuales un $59,7 \%$ fueron pacientes de 6 a 8 años y un $40,3 \%$ pacientes de 9 a 12 años (tabla 1 ).

TABLA 1. Distribución dE LA MUESTRA EVALUADA

\begin{tabular}{|l|l|l|l|l|l|}
\hline Edad & N & $\%$ & Sexo & $n$ & $\%$ \\
\hline 6 a 8 años & 77 & 59,7 & Femenino & 69 & 53,5 \\
\hline 9 a 12 años & 52 & 40,3 & Masculino & 60 & 46,5 \\
\hline Total & 129 & 100,0 & Total & 129 & 100,0 \\
\hline
\end{tabular}

Tabla 2. Prevalencia de caries dental con Relación a La edad

\begin{tabular}{|c|c|c|c|c|c|c|}
\hline \multirow{3}{*}{ Caries dental } & \multicolumn{4}{|c|}{ Edad } & \multirow{2}{*}{\multicolumn{2}{|c|}{ Total }} \\
\hline & \multicolumn{2}{|c|}{6 a 8 años } & \multicolumn{2}{|c|}{9 a 12 años } & & \\
\hline & $\mathrm{n}$ & $\%$ & $\mathrm{n}$ & $\%$ & $\mathrm{n}$ & $\%$ \\
\hline \multirow[t]{2}{*}{ Con caries } & \multirow[t]{2}{*}{57} & \multirow[t]{2}{*}{44,2} & \multirow[t]{2}{*}{53} & \multirow[t]{2}{*}{41} & \multicolumn{2}{|r|}{110} \\
\hline & & & & & \multicolumn{2}{|r|}{85,3} \\
\hline Sin caries & 9 & 7 & 10 & 7,8 & 19 & 14,7 \\
\hline \multirow[t]{2}{*}{ Total } & \multirow[t]{2}{*}{66} & \multirow[t]{2}{*}{51,2} & \multirow[t]{2}{*}{63} & \multirow[t]{2}{*}{48,8} & & 129 \\
\hline & & & & & & 100 \\
\hline
\end{tabular}

Prueba $\mathrm{Xi2}=0,128 \quad \mathrm{p}=0,720$ 
Tabla 3. Prevalencia de caries dental con relación al gÉnero

\begin{tabular}{|c|c|c|c|c|c|c|}
\hline \multicolumn{7}{|c|}{ Género } \\
\hline \multirow[t]{2}{*}{ Caries dental } & \multicolumn{2}{|c|}{ Femenino } & \multicolumn{2}{|c|}{ Masculino } & \multicolumn{2}{|c|}{ Total } \\
\hline & $n$ & $\%$ & $\mathrm{~N}$ & $\%$ & $n$ & $\%$ \\
\hline Con caries & 59 & 45,7 & 51 & 39,5 & 110 & 85,3 \\
\hline Sin caries & 10 & 7,8 & 9 & 7 & 19 & 14,7 \\
\hline Total & 69 & 53,5 & 60 & 46,5 & 129 & 100 \\
\hline Prueba Xi2 = 0,07 & & 935 & & & & \\
\hline
\end{tabular}

TABLa 4. ReLAción ENTRE PH SALIVAL Y CARIES DeNTAL

\begin{tabular}{l|c|c|c|c|c|c|} 
& \multicolumn{5}{|c}{ Caries dental } \\
pH Salival & \multicolumn{2}{|c|}{ Presente } & \multicolumn{2}{|c}{ Ausente } & \multicolumn{2}{c}{ Total } \\
& $\mathrm{N}$ & $\%$ & $\mathrm{~N}$ & $\%$ & $\mathrm{n}$ & $\%$ \\
\hline Ácido & 68 & 52,7 & 3 & 2,3 & 71 & $55 \%$ \\
\hline Neutro & 37 & 28,7 & 16 & 12,4 & 53 & 41,1 \\
\hline Alcalino & 5 & 3,9 & 0 & 0 & 5 & 3,9 \\
\hline Total & 110 & 85,3 & 19 & 14,7 & 129 & 100 \\
\hline Prueba Xi2 $=17,186$ & $\mathrm{p}<0,001$ & & &
\end{tabular}

Se encontró una prevalencia de caries dental del $85,3 \%$ en escolares entre los 6 y 12 años. La prevalencia de caries dental en los niños de 6 a 8 años fue del $44,2 \%$ y del $41 \%$ en los niños de 9 a 12 años, respectivamente (tabla 2).

La prevalencia de caries según el género en la población evaluada fue del $45,7 \%$ en niñas y el $39,5 \%$ en varones. No se encontró asociación estadísticamente significativa entre el género y la prevalencia de caries dental, $p=0,935$ (tabla 3).

Finalmente, al analizar la asociación entre $\mathrm{pH}$ salival y caries dental, se determinó que el $55 \%$ de los pacientes tuvieron un $\mathrm{pH}$ salival ácido, mientras que el $41,1 \%$ presentaron un $\mathrm{pH}$ salival neutro y el $3,9 \%$, un $\mathrm{pH}$ salival alcalino. Se encontró asociación significativa, $\mathrm{p}<$ 0,001 (tabla 4).

Vale resaltar que, de los 71 pacientes con un pH salival ácido, 68 presentaban caries dental, mientras que, de los 53 pacientes con un $\mathrm{pH}$ neutro, solo 37 presentaron caries. En el caso de los 5 pacientes con $\mathrm{pH}$ alcalino, todos presentaron caries dental. A partir de los resultados obtenidos, se puede afirmar que existe una fuerte correlación entre el $\mathrm{pH}$ salival y la prevalencia de caries (tabla 4).

\section{DISCUSIÓN}

El estudio reunió un total de 129 niños de la institución educativa San Gabriel, entre los 6 y 12 años, según los criterios de selección especificados. El tamaño de la muestra evaluada fue algo reducido, pero aun así es relevante para conocer el estado de salud oral de esta población. Se determinó una prevalencia de caries del $85.3 \%$, resultado que se encuentra entre los promedios referenciales previamente establecidos en el Perú. Un estudio de prevalencia realizado por Heredia et al. evaluó a 2482 niños y mostró una prevalencia de caries del 91,5\% $\left({ }^{20}\right)$. De modo similar, Flores y Montenegro encontraron una prevalencia del $89,6 \%$ en la misma población $\left({ }^{21}\right)$. El más reciente estudio epidemiológico a nivel nacional concluyó que, en este grupo etario, la prevalencia 
de caries es del $90.4 \%$, además de resaltar que el CPO-D a los 12 años es de aproximadamente 6 , lo que nos ubica, según la Organización Panamericana de la Salud (OPS), como un país en estado de emergencia ${ }^{(22)}$.

A diferencia de resultados hallados en recientes estudios, no se encontró asociación estadísticamente significativa entre la caries dental y la edad. Agurto y Rosario relatan que esta patología necesita de cierto margen de tiempo para su aparición y propagación $\left({ }^{23}\right)$. Ya que el grupo etario entre 5 y 8 años presenta mayor cantidad de piezas deciduas debido a la secuencia de erupción dentaria, es lógico que estas presenten mayor prevalencia de caries pues han sido expuestas a la cavidad oral por mayor tiempo $\left({ }^{24}\right)$. Los hallazgos de Hernández et al. van en la misma línea ${ }^{(25)}$. Otros investigadores, como Mattos Vela, han encontrado asociación estadísticamente significativa en menores de 6 años con pobre higiene bucal.

Una buena higiene es imprescindible para evitar la aparición de caries sin importar el rango etario. Sin embargo, es en esta etapa que se da la erupción de la primera molar permanente, la cual es determinante en el desarrollo del sistema estomatognático. Es por estos motivos que vale la pena resaltar la importancia de realizar acciones preventivas en este grupo etario. Diversos estudios pregonan lo mismo y destacan la importancia de enseñar una correcta técnica de cepillado dental $(26,27)$.

En cuanto al sexo, no se halló evidencia que lo relacione a la prevalencia de caries, pero cabe mencionar que estos resultados se contrastan con aquellos reportados por Caballero et al., quienes hallaron que los varones presentan mayor prevalencia de caries. Por otra parte, existen estudios que plantean que las niñas ostentan mayor prevalencia dado que son más proclives a fluctuaciones hormonales y su secreción salival es menor $\left({ }^{24}\right)$.

Otro factor que contribuye a la aparición de la caries dental es la presencia de una dieta cariogénica $\left({ }^{28}\right)$. La cariogenicidad de un alimento es más que su contenido de carbohidratos. Tal y como explica Gonzáles Sanz, "hay que considerar varios factores entre los cuales figuran: características físicas del alimento, solubilidad, retención, capacidad para estimular el flujo salival, frecuencia, horario de consumo y tiempo de permanencia en la boca". Otros investigadores resaltan la importancia de comprobar la presencia de apiñamiento dentario. Se dice que a los escolares con esta mal oclusión se les imposibilita realizar una adecuada higiene oral, lo que produce acumulación de placa bacteriana, conformada mayormente por microorganismos patógenos (29-31).

Mayor et al. aseveran que el aumento de la caries guarda relación con la presencia de varios factores biológicos y sociales, tales como las condiciones socioeconómicas, la ingesta de una dieta cariogénica y la presencia de microorganismos cariogénicos como consecuencia de una mala higiene bucal $\left({ }^{32-34}\right)$. Por ende, existe una mayor prevalencia de caries dental en países en vías de desarrollo, lo cual concuerda con nuestros hallazgos $\left({ }^{35}\right)$. Efectuar comparaciones entre países presenta muchas limitaciones; no obstante, resulta interesante contrastar nuestra experiencia con otras realidades. En una mano tenemos las naciones desarrolladas, como Estados Unidos o Canadá, donde la prevalencia de caries no supera el $20 \%$. Esto es algo que comparten con la mayoría de países desarrollados. En Alemania, por ejemplo, los niños de 8 años presumen una prevalencia del $16,6 \%$; una tasa sorprendentemente baja en comparación con la nuestra $\left({ }^{10}\right)$. En cambio, la situación de 
los países en vías de desarrollo es considerablemente más preocupante. Los niños mexicanos de 6 años presentan una prevalencia mucho más alta, que llega hasta el 59\% $\left({ }^{31}\right)$. Del otro lado del mundo tenemos a los niños filipinos, quienes se asemejan a sus contrapartes peruanos con una prevalencia del $68,2 \%$ $\left.{ }^{(36}\right)$. Lo que llamó la atención fue encontrar que los niños brasileños de edad escolar presentan una prevalencia muy por debajo de la nuestra, con tan solo un $40,1 \%\left({ }^{37}\right)$. En el caso de Ecuador, los niños entre 5 a 10 años presentan una prevalencia del 43,2\% $\left({ }^{9}\right)$. El hecho de que otros países dentro de nuestro continente ostenten una prevalencia de caries dental mucho menor debe llevarnos a la reflexión ya que, dadas las cifras mencionadas, las políticas del Estado sobre el manejo de esta enfermedad han fracasado y requieren ser reformadas.

\section{CONCLUSIONES}

1. Se encontró asociación significativa entre el $\mathrm{pH}$ salival y la prevalencia de caries dental.

2. Se calculó que el $95.8 \%$ de los pacientes con un $\mathrm{pH}$ salival ácido presentaron caries dental.

Contribución del autor: Carlos Alberto Araujo ha participado en la concepción del artículo, la recolección de datos, su redacción y la aprobación de la versión final.

Fuente de financiamiento: Autofinanciada.

Conflictos de interés: El autor declara no tener conflictos de interés de ningún tipo. 


\section{REFERENCIAS BIBLIOGRÁFICAS}

1. Animireddy D, Bekkem TRB, Vallala P, Kotha SB, Ankireddy S, Mohammad N. Evaluation of $\mathrm{pH}$, buffering capacity, viscosity and flow rate levels of saliva in caries-free, minimal caries and nursing caries children: An in vivo study. Contemp Clin Dent. 2014; 5 (3): 324-8.

2. García Castro L, Tello Guerrero G, Álvaro Ordóñez L, Perona Miguel de Priego G. Caries dental y microbiota. Revisión. Rev Cient Odontol. 2017; 5 (1): 668-78.

3. Jayaraj D, Ganesan S. Salivary $\mathrm{pH}$ and buffering capacity as risk markers for early childhood caries: a clinical study. Int J Clin Pediatr Dent. 2015; 8 (3): 167-71.

4. Pandey P, Reddy V, Rao AP, Saxena A, Chaudhary CP. Estimation of salivary flow rate, $\mathrm{pH}$ buffer capacity, calcium, total protein content and total antioxidant capacity in relation to dental caries severity, age and gender. Contemp Clin Dent. 2015; 6 (1): 65-71.

5. Dawes C, et al. The functions of human saliva: a review sponsored by the World Workshop on Oral Medicine VI. Arch Oral Biol. 2015; 60 (6): 863-74.

6. Opal S, Garg S, Jain J, Walia I. Genetic factor affecting dental caries risk. Aust Dent J. 2015; 60 (1): 2-11.

7. Juárez RP, Celia AC. Rol de la saliva en la homeostasis de la cavidad bucal y como medio de diagnóstico. Rev Dent Chile. 2015; 106 (2): 15-8.

8. Tenuta LMA, Fernández CE, Brandão ACS, Cury JA. Titratable acidity of beverages influences salivary $\mathrm{pH}$ recovery. Braz Oral Res. 2015; 29 (1): 1-6.

9. Lara Rojas A, Chuquimarca Páucar BR. Prevalencia de caries dental y su relación con el $\mathrm{pH}$ salival en niños y adolescentes con discapacidad intelectual. Dom Cien. 2017; 3 (1): 474-87.

10. Grund, et al. Clinical consequences of untreated dental caries in German 5- and 8-year-olds. BMC Oral Health 2015; 15 (140): 1-11.

11. Kilic $\mathrm{G}$, Cetin $\mathrm{M}$, Ellidokuz $\mathrm{H}$. The relationship of salivary flow rate and salivary $\mathrm{pH}$ on dental caries in children. J Pediatr Res. 2015; 2 (2): 87-91.

12. Singh S, Sharma A, Sood PB, Sood A, Zaidi I, Sinha A. Saliva as a prediction tool for dental caries: an in vivo study. J Oral Biol Craniofac Res. 2015; 5 (2): 59-64.

13. Zhou J, Jiang N, Wang Z, Li L, Zhang J, Ma R, et al. Influences of $\mathrm{pH}$ and iron concentration on the salivary microbiome in individual humans with and without caries. Appl Environ Microbiol. 2017; 83 (4): 2412-6.

14. Li-Hui W, Chuan-Quan L, Long Y, Ru-Liu L, Long-Hui C, Wei-Wen C. Gender differences in the saliva of young healthy subjects before and after citric acid stimulation. Clin Chim Acta. 2016; 460: 142-5.

15. Kianoush N, Adler CJ, Nguyen K-AT, Browne GV, Simonian M, Hunter N. Bacterial profile of dentine caries and the impact of $\mathrm{pH}$ on bacterial population diversity. PLoS ONE. 2014; 9 (3): 92940. 
16. Mohammed HK, Anjana G, Zareena MA, Sunil EA. Antioxidant capacity of saliva: effect on onset and progression of dental caries. Oral Maxillofac Pathol J. 2017; 8 (1): 19-22.

17. Yildiz G, Ermis RB, Calapoglu NS, Celik EU, Türel GY. Gene-environment interactions in the etiology of dental caries. J Dent Res. 2016; 95 (1): 74-9.

18. Prabhakar A, Dodawad $\mathrm{R}$, Os $\mathrm{R}$. Evaluation of flow rate, $\mathrm{pH}$, buffering capacity, calcium, total protein and total antioxidant levels of saliva in caries free and caries active children - an in vivo study. Int J Clin Pediatr Dent. $2009 ; 2$ (1): 9-12.

19. Reis N, Oom MS, Vicente AF, Amorim A, Delgado J, Godinho C. Influence of intensive training on salivary flow, on salivary $\mathrm{pH}$ and on salivary lactate concentration: consequences for oral health. CiiEM; 2015.

20. Heredia C, Alva F. Relación entre la prevalencia de caries dental y desnutrición crónica en niños de 5 a 12 años de edad. Rev Estomatol Herediana 2005;15 (2): 124-7.

21. Flores M, Montenegro B. Relación entre la frecuencia diaria de consumo de azúcares extrínsecos y la prevalencia de caries dental. Rev Estomatol Herediana 2005; 15 (1): 36-9.

22. Minsa; 2017 [actualizado 14 de Mar 2017; citado 20 abr 2017] Disponible en: http:// www.minsa.gob.pe/portalweb/06prevencion/prevencion_2.asp?sub5=13

23. Caballero García C, Enríquez G, García Rupaya C. Relación entre la experiencia de caries dental e higiene bucal en escolares de la provincia de Sechura-Piura en el año 2010. Rev Estomatol Herediana. 2012; 22 (1): 16-9.

24. Morales Sosa N. Factores de riesgo en la aparición de la caries dental. Revisión bibliográfica. 2015.

25. Agurto A, Rosario A. Prevalencia de caries dental en niños de 6 a 12 años de edad atendidos en la Clínica Pediátrica de la Universidad Alas Peruanas utilizando los criterios de Icdas II. [Tesis de grado]. Lima: Universidad Alas Peruanas; 2010.

26. Hernández Suárez A, Espeso Nápoles N, Reyes Obediente F, Rodríguez Borges L. Intervención educativo-curativa para la prevención de caries dental en niños de cinco a 12 años. AMC. 2015; 14 (6): 1-9.

27. Mattos Vela MA. Factores socioeconómicos y de comportamiento relacionados con caries dental en escolares del distrito de La Molina, Lima, Perú. Rev Estomatol Herediana. 2010; 20 (1): 25-32.

28. Gispert Abreu E, Herrera Nordet M, Felipe Lazo I. Prevención indirecta de caries en la temprana infancia. Rev Cubana Estomatol. 2006; 43 (4): 61-83.

29. González Sanz AM, González Nieto BA, González Nieto E. Salud dental: relación entre la caries dental y el consumo de alimentos. Nut Hospital. 2013; 28 (4).

30. Sánchez L, Sáenz P, Alfaro M, Carmen Osorno C. Comportamiento del apiñamiento, gingivitis, higiene oral, caries, flujo salival y bacterias en escolares de 8 y 10 años. Rev ADM. 2013; 70 (2): 91-7. 
31. Sánchez Pérez L, Alanís Tavira J, Vera Hermosillo H, Rodríguez Gurza ME, Arjona Serrano J, Sáenz Martínez LP. Factores de riesgo para caries en escolares rurales y urbanos de Yucatán. Rev Ciencias Clínicas. 2013; 14 (1): 3-11.

32. Mayor Hernández F, Pérez Quiñones JA, Cid Rodríguez MC, Martínez Brito I, Martínez Abreu J, Moure Ibarra MD. La caries dental y su interrelación con algunos factores sociales. Artículo de revisión. Rev Med Electrón. 2014 [citado 22 Oct 2015]; 36 (3).

33. Christenser LB, Twetman S, Sundby A. Oral health in children and adolescents with diferent socio/cultural and socio/economic backgrounds. Acta Odontol Scand. 2010; 68 (1): 34-42.

34. Psoter WJ, Pendrys DG, Morse DE, Zhang H, Mayne ST. Associations of ethnicity/race and socioeconomic status with early childhood caries patterns. J Public Health Dent. 2006; 66 (1): 23-9.

35. Prakash P, Subramaniam P, Durgesh BH, Konde S. Prevalence of early childhood caries and associated risk factors in preschool children of urban Bangalore, India: a cross/sectional study. Eur J Dent. 2012; 6: 114-52.

36. Yabao RN, et al. Prevalence of dental caries and sugar consumption among 6-12y-old schoolchildren in La Trinidad, Benguet, Philippines. Eur J Clin Nutr. 2005; 59: 1429-38.

37. Almeida RF, Leal SC, Mendoça JGA, Hilgert LA, Ribeiro APD. Oral health and school performance in a group of schoolchildren from the Federal District, Brazil. J Public Health Dent. 2018; 78 (4): 306-12. 